\title{
BRONQUIOLITIS RESPIRATORIA Y NEUMONÍA DESCAMATIVA ASOCIADA A FUNGEMIA POR TRICHOSPORON ASAHII EN INMUNOCOMPETENTE:
}

\section{A PROPÓSITO DE UN CASO}

\author{
ALIRIO R. BASTIDAS MD. MSc*1, JAIME A. PANTOJA MD. MSc. PhD.(c) ${ }^{2}$, \\ MARÍA A. MUNAR MD', \\ LUIS F. GIRALDO MD. FCCP. PhD. ${ }^{2}$, JAMES YURGAKI MD.3 \\ ${ }^{1}$ Servicio de Neumología y Terapia respiratoria, Clínica Universidad de La Sabana, \\ Chía, Cundinamarca, Colombia. \\ ${ }^{2}$ Facultad de Medicina, Universidad de La Sabana, Chía, Cundinamarca, Colombia. \\ ${ }^{3}$ Facultad de Medicina, Universidad Militar Nueva Granada, Hospital Militar Central, \\ Bogotá DC, Colombia. \\ *1Correspondencia: Alirio Rodrigo Bastidas Goyes; alirio.bastidas@clinicaunisabana.edu.co \\ Recibido: Mayo 15 de 2015 \\ Aceptado: Octubre 28 de 2015

\section{Resumen}

Trichosporon asahii es un hongo patógeno emergente reportado en la literatura médica principalmente en pacientes inmunocomprometidos. No obstante, el presente caso es inusual debido a que se trata de un paciente adulto joven inmunocompetente que presentó fungemia por T. asahii y al mismo tiempo desarrolló insuficiencia respiratoria aguda por bronquiolitis respiratoria y neumonía descamativa, la cual resolvió posterior al tratamiento antimicótico instaurado, soporte ventilatorio y vigilancia en Unidad de Cuidado Intesivo (UCI).

Palabras claves: Inmunocompetencia, Fungemia, Trichosporon asahii, Neumonía descamativa. 


\title{
RESPIRATORY BRONCHIOLITIS AND DESQUAMATIVE INTERSTITIAL PNEUMONIA ASSOCIATED WITH FUNGEMIA BY TRICHOSPORON ASAHII IN IMMUNOCOMPETENT:
}

\section{A CASE REPORT}

\begin{abstract}
Trichosporon asahii is an emerging fungal pathogen reported in the medical literature mainly in immunologically compromised patients. However, this case is unusual because is a young immunocompetent patient who developed fungemia by $T$. asahii simultaneously with acute respiratory failure, respiratory bronchiolitis and desquamative interstitial pneumonia, who responded satisfactorily to ventilatory support and antifungal therapy.
\end{abstract}

Keywords: Immunocompetence, Fungemia, Trichosporon asahii, Desquamative pneumonia.

\section{BRONQUIOLITE RESPIRATÓRIA E PNEUMONIA INTERSTICIAL DESQUAMATIVA ASSOCIADA A FUNGÔMIA POR TRICHOSPORON ASAHII EM IMUNO-COMPONENTE: \\ UM RELATO DE CASO}

\begin{abstract}
Resumo
Trichosporon asahii é um patógeno fúngico emergente relatado na literatura médica principalmente em pacientes imunologicamente comprometidos. No entanto, este caso é incomum porque é um jovem imunocompetente que desenvolveu fungemia por $T$. asahii simultaneamente com insuficiência respiratória aguda, bronquiolite respiratória e pneumonia intersticial descamativa, que responderam satisfatoriamente ao suporte ventilatório e à terapia antifúngica.
\end{abstract}

Palavras-chave: Imunocompetência, Fungemia, Trichosporon asahii, Pneumonia descamativa. 


\section{Introducción}

Los Trichosporon son reconocidos en la actualidad como organismos levaduriformes, los cuales pueden estar presentes en la piel humana como flora normal, igualmente pueden ser aislados en las vías respiratorias y en el tracto gastrointestinal (1). La especie Trichosporon asahii en un $68 \%$ de los casos, es uno de los principales agentes causales de infecciones sistémicas emergentes por hongos filamentosos (2). Sin embargo, cuando hacemos referencia a los agentes causales de fungemia, puede llegar a ocupar el segundo o tercer lugar entre la lista de los principales microorganismos productores de la enfermedad (3-5).

Usualmente ésta especie ocasiona infecciones dermatológicas no fatales en pacientes inmunocompetentes. No obstante, en aquellas personas que tienen algún compromiso inmunológico puede llegar a generar una micosis sistémica que puede llegar a ser fatal (6).

\section{Presentación del caso}

Hombre de 19 años de edad, soldado regular de profesión, procedente de una zona selvática del departamento de Mitú, Colombia. Consultó refiriendo síntomas de 7 días de evolución que iniciaron con: astenia, adinamia, dolor articular, mialgias, fiebre y deposiciones diarreicas. Durante las últimas 48 horas presentó deterioro clínico respiratorio asociado a tos seca y disnea severa. Fue atendido inicialmente en el hospital regional donde le suministraron medidas de soporte ventilatorio y lo remitieron a una UCI de un centro de mayor complejidad.

Paciente previamente sano, sin contacto con sintomáticos respiratorios, sin antecedentes quirúrgicos o transfusionales, consumidor ocasional de licor y fumador de menos de 1 paquete/año de cigarrillo; no había historia de alergias ni de enfermedades de transmisión sexual.

Al examen físico de ingreso en la Unidad de Cuidado Intensivo el paciente se encontraba con requerimiento de soporte ventilatorio y vasopresor, los signos vitales evidenciaban: TA: 108/47 mmHg, FC: 115 latidos por minuto, FR: 16 respiraciones por minuto, $\mathrm{T}^{\circ}: 38,3{ }^{\circ} \mathrm{C}$, saturación de oxígeno de 94\%, con $\mathrm{FIO}_{2}: 60 \%$.

Como otros hallazgos al examen físico se encontró: mucosa oral húmeda, faringe sin lesiones y el cuello sin evidencia de adenomegalias, la expansibilidad del tórax se encontraba conservada y a la auscultación pulmonar había estertores gruesos diseminados en ambos campos pulmonares, ruidos cardiacos rítmicos, taquicárdicos, sin auscultarse soplos. No se evidenció hepatomegalia ni esplenomegalia, las extremidades no presentaban edemas y el examen neurológico no tenía déficit motor ni sensitivo aparente.

El cuadro hemático mostró anemia, leucocitosis y neutrofilia. (Tabla 1)

Tabla 1. Hemograma ingreso UCI.

\begin{tabular}{|c|c|c|}
\hline Paraclínico & Resultado & Control \\
\hline Leucocitos & $22.000 \mathrm{cel} / \mathrm{ml}$ & $4,5-11,5 \mathrm{cel} / \mathrm{ml}$ \\
\hline Neutrófilos & $83 \%$ & $55-70 \%$ \\
\hline Linfocitos & $10 \%$ & $17-45 \%$ \\
\hline Eosinófilos & $3 \%$ & $1-4 \%$ \\
\hline Monocitos & $4 \%$ & $2-8 \%$ \\
\hline Plaquetas & $247.000 \mathrm{cel} / \mathrm{ml}$ & $150-450 \mathrm{cel} / \mathrm{ml}$ \\
\hline Hemoglobina & $11.7 \mathrm{gr} / \mathrm{dl}$ & $13,5-18,0 \mathrm{gr} / \mathrm{dl}$ \\
\hline Hematocrito & $30.5 \%$ & $42-52 \%$ \\
\hline
\end{tabular}

Tenía una ligera elevación de: nitrógeno uréico, transaminasas y procalcitonina, la proteína $\mathrm{C}$ reactiva estaba severamente elevada (Tabla 2 )

Tabla 2. Química sanguínea ingreso UCI.

\begin{tabular}{|l|c|c|}
\hline \multicolumn{1}{|c|}{ Paraclínicos } & Resultado & Control \\
\hline BUN & $26 \mathrm{mg} / \mathrm{dl}$ & $5-20 \mathrm{mg} / \mathrm{dl}$ \\
\hline Creatinina & $1,07 \mathrm{mg} / \mathrm{dl}$ & $<1,5 \mathrm{mg} / \mathrm{dl}$ \\
\hline Sodio & $141 \mathrm{mEq} / \mathrm{L}$ & $135-145 \mathrm{mEq} / \mathrm{L}$ \\
\hline Potasio & $5,1 \mathrm{mEq} / \mathrm{L}$ & $3,4-4,8 \mathrm{mEq} / \mathrm{L}$ \\
\hline Cloro & $110 \mathrm{mEq} / \mathrm{L}$ & $100-108 \mathrm{mEq} / \mathrm{L}$ \\
\hline PCR & $384 \mathrm{mEq} / \mathrm{L}$ & $0-12 \mathrm{mEq} / \mathrm{L}$ \\
\hline AST/TGO & $77 \mathrm{U} / \mathrm{L}$ & $32 \mathrm{U} / \mathrm{L}$ \\
\hline ALT/TGP & $53 \mathrm{U} / \mathrm{L}$ & $53 \mathrm{U} / \mathrm{L}$ \\
\hline Procalcitonina & $0,6 \mathrm{ng} / \mathrm{ml}$ & $<0,5 \mathrm{ng} / \mathrm{ml}$ \\
\hline Glicemia & $146 \mathrm{meq} / \mathrm{L}$ & ayunas:70-100 mg/dL \\
\hline
\end{tabular}

La radiografía $(\mathrm{Rx})$ de tórax mostró infiltrados nodulares en ambos campos pulmonares, sin derrame pleural ni aumento del pedículo vascular (Figura 1). 


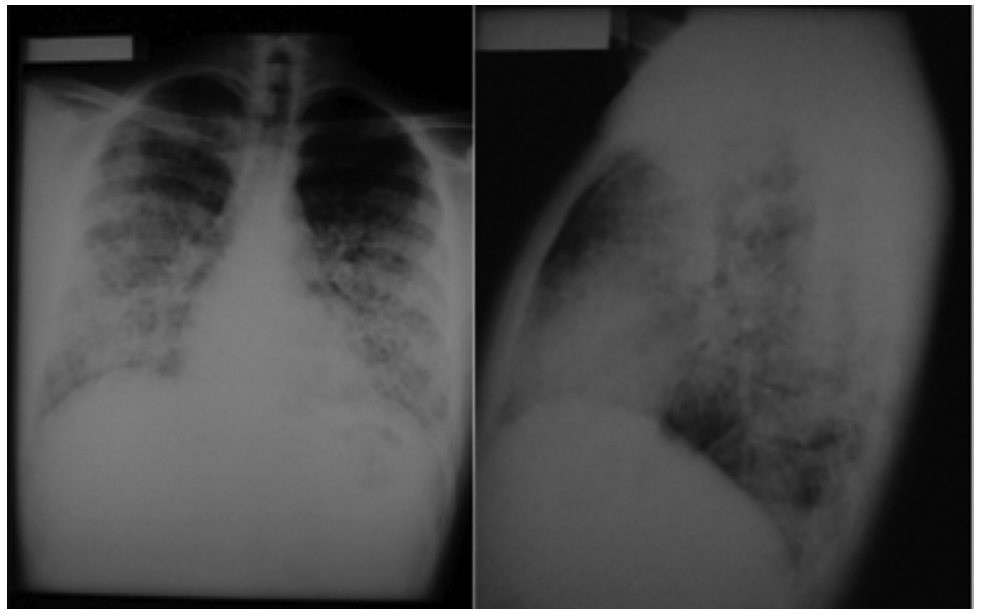

Figura 1. Radiografía de Tórax PA y Lat.

La tomografía axial computarizada (TAC) de tórax mostró infiltrados mixtos: ocupación alveolar principalmente en las regiones basales-posteriores e infiltrados nodulares especialmente hacia las regiones anteriores (Figura 2).

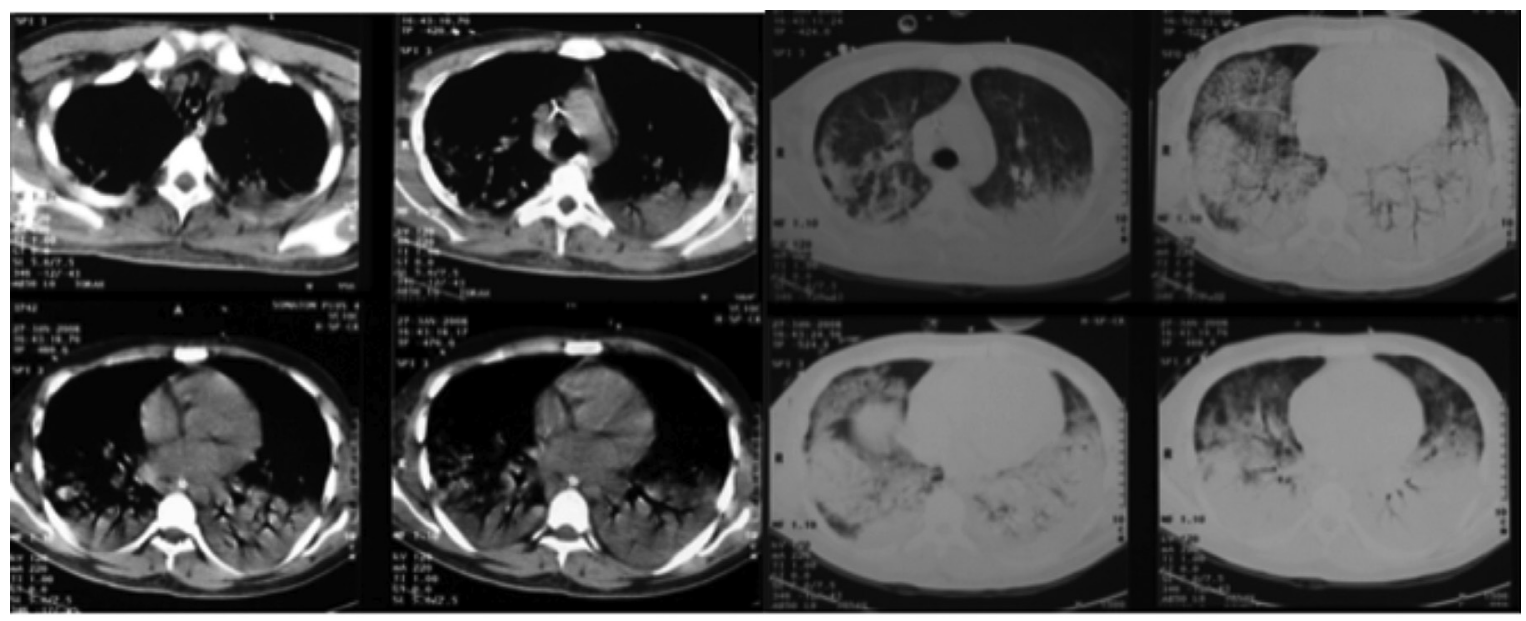

Figura 2: Tomografía Axial Computarizada de Tórax.

Durante la estancia en UCI se practicó una fibrobroncoscopia donde se apreció un árbol traqueobronquial sin lesiones; en el lavado broncoalveolar (BAL) para estudio de gérmenes comunes, micobacterias, hongos y recuento diferencial de células se observó: células epiteliales con cambios reactivos acompañados de histiocitos, linfocitos y polimorfonucleares neutrófilos; recuento celular en 500 células por ml, con diferencial de: PMN neutrofilos 40\%, linfocitos: $10 \%$ y células epiteliales $10 \%$. La coloración hierro fue negativa para hemosiderófagos, citología negativa para malignidad, las coloraciones de: PAS, Ziehl Neelsen (ZN) y plata fueron negativas; el cultivo del BAL fue negativo a las 48 y 72 horas. El ecocardiograma se mostró una fracción de eyección del ventrículo izquierdo del $56 \%$, sin alteraciones valvulares ni vegetaciones.

Durante las primeras 48 horas de su ingreso se reportó crecimiento de Trichosporon asahii en los hemocultivos (Figura 3), se inició tratamiento con Anfotericina B y Voriconazol teniendo en cuenta dicha positividad, igualmente se realizó ELISA para VIH con resultado negativo, como posible causa de inmunocompromiso dados los antecedentes laborales y la edad del paciente. 


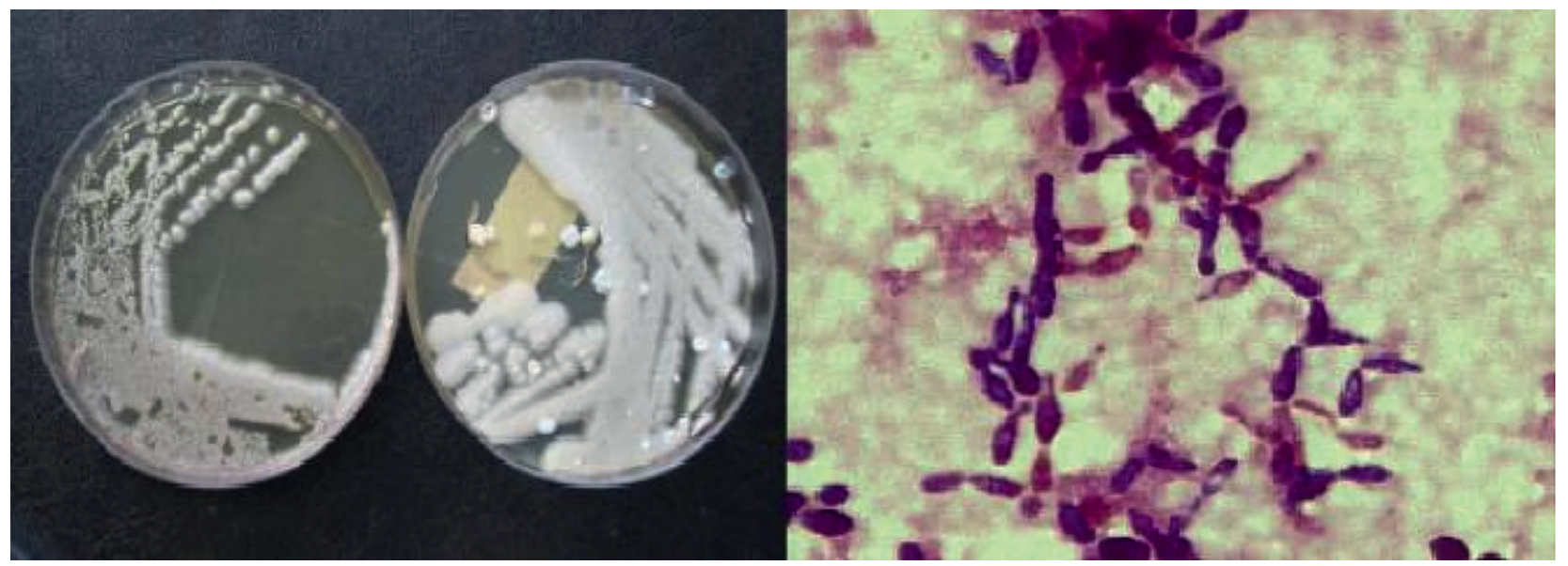

Figura 3. Crecimiento del Hongo en agar Sabouraud y hemocultivos.

Durante el curso clínico inmediato, el paciente presentó una evolución irregular, con mayor deterioro del patrón respiratorio y persistencia de infiltrados pulmonares, por lo cual fue llevado a biopsia pulmonar abierta, cuyo estudio histopatológico mostró bronquiolitis respiratoria con patrón de neumonía intersticial descamativa (Figu- ra 4). Igualmente, se le practicaron tinciones de: tricromo, azul de prusia y PAS, las cuales fueron negativas, asimismo la inmunohistoquímica con CD1 y CD64 para descartar patología de origen tumoral asociado a histiocitos fueron negativas.

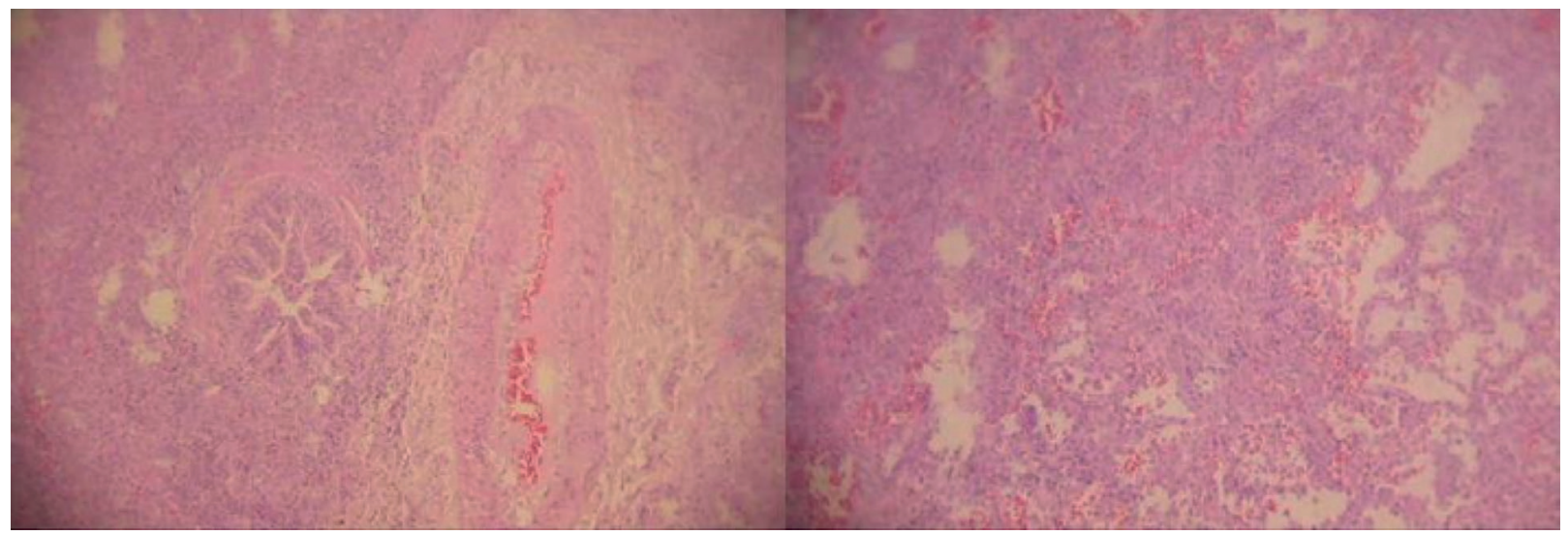

Figura 4. Histología biopsia pulmonar abierta, tinción hematoxilina-eosina.

El paciente presentó deterioro de la función renal, lo que obligó a la suspensión de la Anfotericina B y se decidió continuar monoterapia con Voriconazol. Dos semanas después del ingreso, tuvo recuperación paulatina de los índices de oxigenación, pudo retirarse el soporte vasopresor y finalmente se retiró la ventilación mecánica. El paciente fue trasladado a salas generales hasta completar 4 semanas de tratamiento con voriconazol, recuperación física y nutricional. Durante la estancia en UCI recibió inicialmente tratamiento antibiótico de am- plio espectro y posteriormente se continuó únicamente con antifúngicos, profilaxis antiembolica, protección gástrica, sedación y líquidos endovenosos, sin terapia esteroidea.

A la salida del hospital se realizaron controles con pruebas de función pulmonar a los 6 meses de la hospitalización, donde se evidenció una alteración ventilatoria restrictiva leve y una disminución leve en la difusión de monóxido de carbono (Tabla 3). 
Tabla 3. Prueba de función pulmonar control a los 6 meses del egreso.

\begin{tabular}{|l|c|c|c|c|c|}
\hline & Predicho & Hallado & \% Predicho & Post B2 & \% Cambio \\
\hline CVF (Litros) & 5,20 & 2,53 & 48,62 & 2,43 & $-3,95$ \\
\hline VEF1 (Litros) & 4,43 & 2,49 & 56,26 & 2,42 & $-2,81$ \\
\hline VEF1/CVF (\%) & 85,37 & 98,42 & & 99,59 & 1,19 \\
\hline FEF 25-75 (L/sec) & 4,90 & 3,85 & 78,58 & 4,97 & 29,09 \\
\hline PEF (L/sec) & 9,42 & 5,76 & 61,17 & 6,86 & 19,10 \\
\hline
\end{tabular}

Lectura: alteración ventilatoria restrictiva.

La tomografía axial computarizada de tórax a los 6 meses de la hospitalización mostró resolución de los infiltrados pulmonares (Figura 5).

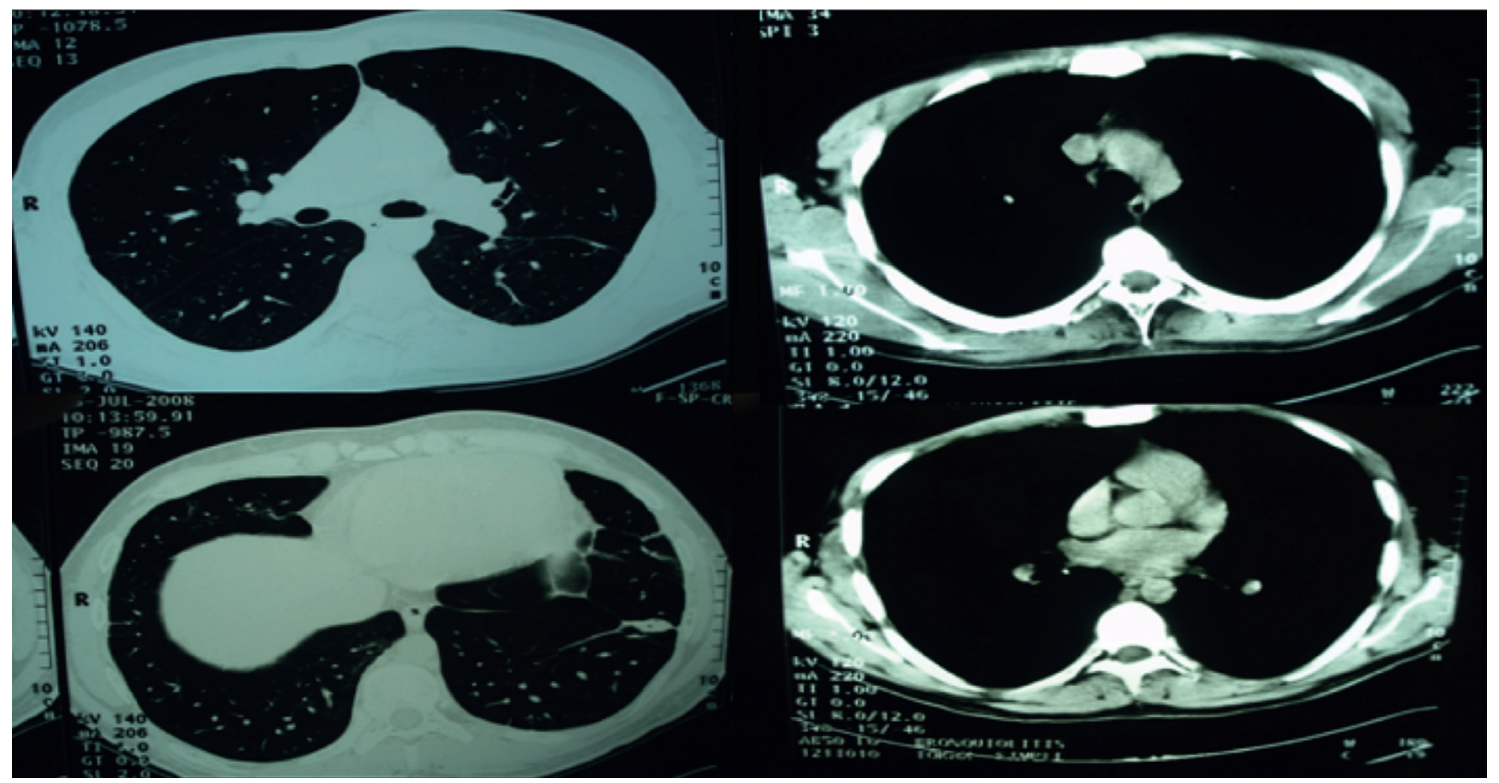

Figura 5. Tomografía Axial Computarizada de tórax control a los 6 meses.

\section{Discusión}

Los Trichosporon spp. son hongos levaduriformes (7) que se pueden encontrar en el suelo, agua, plantas, colonizando dispositivos endoscópicos y piel humana (8). De acuerdo a sus características genéticas, morfológicas y bioquímicas se reconocen 6 especies asociadas a enfermedad en humanos: $T$. cutaneum y $T$. asteroides asociado a infecciones cutáneas superficiales, $T$. ovoides y $T$. inkin agentes etiológicos de la piedra blanca en cabello y vello del pubis, T. asahii y $T$. mucoides que según la literatura son agentes de enfermedad invasiva especialmente en huéspedes inmunosuprimidos (9).
Su puerta de entrada puede ser la piel, el tracto respiratorio, el tracto gastrointestinal y por contaminación de catéteres, generando una diseminación en el organismo con: fungemia, endocarditis, falla renal, lesiones cutáneas y coriorretinitis principalmente en pacientes inmunosuprimidos. Su inhalación se ha relacionado con neumonía $(10,11)$ y en Japón el estudio de estas levaduras se han identificado componentes de pared desencadenantes de la neumonitis de hipersensibilidad del verano $(12,13)$. 
Por otra parte, la bronquiolitis respiratoria con neumonía descamativa es un tipo de enfermedad intersticial pulmonar que se caracteriza por la acumulación de macrófagos en los espacios alveolares, asociado a fibrosis e inflamación intersticial. Se relaciona con el hábito de fumar en más del $90 \%$ de los casos y su evolución se caracteriza por ser subaguda o crónica, sin ser una causa frecuente de insuficiencia respiratoria $(14,15)$.

Los casos de T. asahii en pacientes inmunocompententes son infrecuentes, sin embargo, se debe tener en cuenta como parte de las infecciones como germen emergente (16). En nuestro paciente se desarrolló simultáneamente una fungemia por Trichosporon asahii y un cuadro de insuficiencia respiratoria con infiltrados pulmonares secundarios a bronquiolitis respiratoria con neumonía intersticial descamativa. Si bien, no es evidencia médica suficiente para afirmar una causalidad, si se debe tener en cuenta una posible relación entre la infección fúngica y este patrón histopatológico pulmonar no reportado antes (10).

Aunque no podemos descartar completamente que el tabaquismo haya contribuido a la etiología de la enfermedad intersticial y la insuficiencia respiratoria aguda de este paciente $(15,17)$, pensamos que el factor etiológico más probable de su grave compromiso respiratorio fue la fungemia por $T$. asahii, debido a que el cuadro apareció durante la misma y se resolvió completamente con el tratamiento farmacológico, adicionalmente si tenemos en cuenta que el antecedente de exposición del paciente al tabaquismo fue muy inferior a 10 paquetes/año, que es el índice habitual por encima del cual suele presentarse la enfermedad pulmonar intersticial (18-21).

Finalmente, la alteración residual en las pruebas de función pulmonar de control en este paciente es consistente con lo esperado para este tipo de casos críticos atendidos en la unidad de cuidados intensivos con compromiso ventilatoria severo.

\section{Conclusiones}

El Trichosporon asahii puede comprometer pacientes inmnocompetentes y posiblemente agravar enfermedades pulmonares, el manejo antimicótico oportuno y la terapia de soporte son decisivos para una óptima recuperación. En el caso del paciente que presentamos, consideramos a manera de posible factor de riego causal para adquirir la infección, la exposición continua del paciente a un ambiente selvático - entiéndase - húmedo y con condiciones sanitarias adversas que pueden poner en entredicho los mecanismos fisiológicos de defensa del hombre ante este tipo de microorganismos.

No obstante, reconocemos que hace falta estudios de mayor envergadura para afirmar al $T$. asahii como un agente causal claro de bronquiolitis respiratoria y neumonía descamativa, aunque la coexistencia de estos dos eventos en nuestro paciente abre el interrogante al futuro para ulteriores estudios que se propongan establecer dicha conexión.

\section{Instituciones donde se llevó a cabo el trabajo}

Clínica Universidad de La Sabana

Universidad de La Sabana

Hospital Militar Central

\section{Aporte de los autores al documento}

El doctor Bastidas intervino en el diagnóstico y manejo del paciente, redacción de la historia clínica, consecución de las imágenes, discusión y revisión del documento final.

Los doctores Pantoja, Munar y Giraldo intervinieron en la redacción de la historia clínica, discusión, redacción y revisión del documento final.

El doctor Yurgaki intervino en el diagnóstico, manejo del paciente y redacción de la historia clínica.

\section{Conflictos de intereses y financiación}

Los autores no tienen conflictos de intereses relacionados con la presente publicación.

Esta publicación fue realizada con el apoyo del Hospital Militar Central, de la Clínica Universidad de La Sabana y de la Universidad de La Sabana.

Referencias

1. Suzuki K, Nakase, Kyo T, Kohara T, Sugawara Y, Shibazaki $\mathrm{T}$, et al. Fatal Trichosporon fungemia in patients with hematologic malignancies. European Journal of Haematology. 2010;84(5):441-447.

2. Chagas TC, Chaves GM, Melo ASA, Colombo AL. Bloodstream infections due to Trichosporon spp.: Species distribution, Trichosporon asahii genotypes determined on the basis of ribosomal DNA intergenic spacer 1 sequencing, and antifugal susceptibility testing. J Clin Microbiol 2009; 47(4):1074-1081.

3. Biasoli MS, Carison D, Chigamer GJ, Parodi R, Greca 
A, Tosello ME, Lique AG, Montero A. Systemic infection caused by Trichosporon asahii in a patient with liver transplant. Med Mycol 2008;46:719-723.

4. Bayramoglu G, Sonmez M, Tosun I, Aydin K, Aydin F. Breakthrough Trichosporon asahii fungenia white receiving caspofungin. Infection. 2008;36:68-70.

5. Mendoza M, Rico ME. Trichosporon spp. as a Causative Agent of Onychomycosis. Kasmera. 2011;39(1):26-30.

6. Rastogi VL, Nirwan PS. Invasive trichosporonosis due to trichosporon asahii in a non-immunocompromised host: a rare case report. Indian Journal of Medical Microbiology, 2007;25(1):59-61.

7. Varkey J, Perfect J. Rare and Emerging Fungal Pulmonary Infections. Semin Respir Crit Care Med. 2008;29:121131.

8. Walsh T.J, Groll A.H, Emerging fungal pathogens: evolving challenges to immunocompromised patients for the twenty-first century. Transplant Infectious Disease. 1999;1(4):247-261.

9. Lacroix $C$, Feuilhade de Chauvin. Infections dues à Trichosporon spp. et à Geotrichum spp. EMC-Maladies Infectieuses. 2005;2:97-104.

10. Zeynep Ceren Karahan Z, Koyuncu E, Dolapci I, Arikan Akan O, Can F, Tekel A. Genotyping of Trichosporon asahii strains isolated from urinary tract infections in a Turkish university hospital. Turk J Med Sci. 2010;40(3):485-493.

11. Erer B, Galimberti M, Lucarelli G, Giardini C, Polchi P, Baronciani D, Gaziev D, Angelucci E, Izzi G. Trichosporon beigelii: a life-threatening pathogen in immunocompromised hosts. Bone Marrow Transplantation. 2000;25(7):745-749.

12. Unoura K, Miyazaki Y, Sumi Y, Tamaoka M, Sugita T, Inase $\mathrm{N}$. Identification of fungal DNA in BALF from patients with home-related hypersensitivity pneumonitis. Respir Med. 2011 Nov;105(11):1696-703.

13. Nakajima M, Sugita T, Mikami Y. Granuloma associated with Trichosporon asahii infection in the lung: Unusual pathological findings and PCR detection of Trichosporon DNA. Med Mycol. 2007;45(7):641-4.
14. Baloira A, Xaubet A, Rodríguez Becerra E, Romero AD, Casanova A, Ancochea J. Desquamative interstitial pneumonia and respiratory bronchiolitis-associated interstitial lung disease: data from the Spanish patient registry. Arch Bronconeumol. 2008;44(9):499-503.

15. Ryu J, Myers J, Capizzi S, Douglas W, Vassallo R, Decker P. Desquamative interstitial pneumonia and respiratory bronchiolitis- associated interstitial lung disease. Chest. 2005; 127; 178-184.

16. Wolf DG, Falk R, Hacham M, Theelen B, Boekhout T, Scorzetti G, Shapiro M, Block C, Salkin IF, Polacheck I. Multidrug-resistant Trichosporon asahii infection of nongranulocytopenic patients in three intensive care units. J Clin Microbiol. 2001;39(12):4420-5.

17. Kawabata Y, Hoshi E, Murai K et al. Smoking-related changes in the background lung of specimens resected for lung cancer: a semiquantitative study with correlation to postoperative course. Histopathology. 2008;53:707714.

18. Vassallo R, Jensen EA, Colby TV, Ryu JH, Douglas WW, Hartman TE, Limper AH. The overlap between respiratory bronchiolitis and desquamative interstitial pneumonia in pulmonary Langerhans cell histiocytosis: high-resolution CT, histologic, and functional correlations. Chest. 2003;124(4):1199-205.

19. Fraig M, Shreesha U, Savici D, Katzenstein AL. Respiratory bronchiolitis: a clinicopathologic study in current smokers, ex-smokers, and never-smokers. Am J Surg Pathol. 2002;26(5):647-53.

20. Park JS, Brown KK, Tuder RM, Hale VA, King Jr TE, Lynch DA. Respiratory bronchiolitis-associated interstitial lung disease: radiologic features with clinical and pathologic correlation. J Comput Assist Tomogr. 2002;26(1):1320.

21. Moon J, du Bois RM, Colby TV, Hansell DM, Nicholson AG. Clinical significance of respiratory bronchiolitis on open lung biopsy and its relationship to smoking related interstitial lung disease. Thorax. 1999;54(11):1009-14. 